\title{
Effects of Driving Situation and Driving Experience on Eye Movements
}

\author{
Yumiko Shinohara*, Yukiko Nishizaki ${ }^{\dagger}$
}

\begin{abstract}
A previous investigation of the effects of familiarity with the driving situation on driver's eye movements assessed differences in eye movements between novice and expert drivers when driving in familiar and unfamiliar situations. The findings indicated that familiarity with the driving situation had a greater influence on expert drivers than on novice drivers. We assessed the validity of those results using number of saccades and gaze position. We found that the mean number of saccades elicited in the unfamiliar driving situation was significantly greater in the expert group than in the novice group. Thus, we confirmed the effects of familiarity with the situation and driving experience on driver's eye movements.
\end{abstract}

Keywords: driving experience, driving in a foreign country, eye movements, saccade.

\section{Introduction}

Interest in automated driving systems has grown with the rapid increase in technology. Although a car accident may be attributable to the driver, vehicle, or environment, about $90 \%$ of accidents are caused by driver-related factors [1][2]. Moreover, about $41 \%$ of driver-related accidents are due to recognition errors (i.e., inattention, internal and external distractions, and inadequate surveillance) [1]. Thus, a major reason for the development of automated vehicles is to increase driving safety and reduce the number of car accidents caused by human error, particularly recognition errors. However, because the development of fully autonomous vehicles will occur in phases, it is necessary to approach automated driving systems in a step-wise progression from "non-automation" to "full automation" [3]. Effective cooperation between the driver and the automated driving system relies on the driver characteristics and the driving situation.

Previous studies have shown that novice drivers have a longer eye-fixation duration than expert drivers and that the fixation location differed between novice and expert drivers [4][5][6]. Novice drivers tend to focus on guardrails to determine the position of their vehicles [4]. When turning into a curve, expert drivers gaze to the inside edge of the lane, whereas novice drivers focus on the area between the center and outside edge of the lane. Furthermore, fixation duration is affected by the spatial extent of the useful field of view; that is, the visual area in which in-

* Graduate School of Information Science, Kyoto Institute of Technology, Kyoto, Japan

$\dagger$ Information and Human Sciences, Kyoto Institute of Technology, Kyoto, Japan 
formation can be extracted in a single fixation without eye or head movements [7]. Increased cognitive workload in a complex traffic environment causes the useful field of view to narrow, thus, decreasing the fixation duration [8]. Several previous studies have found differences in eye movements according to driving experience and/or traffic conditions, such as the type of road or the amount of traffic information [7][8][9].

To develop an automated driving system that takes driver characteristics and driving situation into consideration, Shinohara and Nishizaki [10] focused on individual differences in eye movements while driving. The authors compared total fixation duration in novice and expert drivers during simulated driving tasks in their country and in a foreign country and found that the total fixation duration of expert drivers was shorter during the simulated driving task in the foreign country than in their own country. Moreover, they found that familiarity with the driving situation had a greater influence on expert drivers; thus, the authors concluded that the shorter fixation duration exhibited by expert drivers was due to increased cognitive workload when adapting to a novel situation. Miura [8] investigated the relationship between fixation duration and cognitive workload and found that increased cognitive workload shortened fixation duration. The findings of Shinohara and Nishizaki [10] were consistent with those of Miura [8]. Eye saccades are also correlated with cognitive workload. Harbluk and Noy [11] and Dong et al. [12] reported that increased cognitive workload reduced the number of saccades. Thus, the number of saccades initiated when the cognitive workload is increased should be investigated to confirm and extend the hypothesis that the increased fixation duration is caused by an increase in cognitive workload. Moreover, although Shinohara and Nishizaki [10] defined areas of interest (AOI) and calculated fixation duration in each AOI to indicate the focus of the participants' gaze, the analyses had limitations warranting a more detailed investigation of eye movements.

We evaluated the validity of Shinohara and Nishizaki's findings [10] and analyzed eye movements using saccades and gaze position. The paper is organized as follows. Shinohara and Nishizaki's study is described in Section 2. The results of additional analyses of the number of saccades and gaze position are presented in Section 3. Section 4 provides a summary of our findings and conclusions.

\section{Differences in Eye Movements Between Novice and Expert Drivers when Driving Abroad}

Shinohara and Nishizaki [10] investigated differences in eye movements, particularly fixation duration and location, between novice and expert drivers when driving abroad. Participant eye movements were measured during driving simulations in their own country and in a foreign country. In this section, we review the methods, results, discussion, and conclusions of the study.

\subsection{Methods}

\subsubsection{Participants}

The study included 47 Japanese individuals ( 23 females, 24 males) aged between 21 and 59 years $(M=32.3, S D=12.7)$. The participants were divided into two groups based on driving experience. Participants who had driven a car for more than 2 years (whole years) and at least once per week were deemed expert drivers (11 females, 11 males). The remaining participants were deemed novice drivers ( 12 females, 13 males). 


\subsubsection{Apparatus and Materials}

Eye movements were measured using an eye tracker (X2-60, Tobii, Japan) attached to a 27-inch screen (GW2255, BenQ Corp., Japan). Participants watched one video for practice (on a highway in Canada) and two experiment videos, one made in Osaka, Japan and the other in San Francisco, CA, USA, keeping their hands on the wheel (T80, Thrustmaster). Figure 1 shows the experiment room. The practice video did not show any cultural characteristics, such as types of lanes or right- or left-hand traffic. The two videos used for measuring eye movements in the experiment were taken on clear days in October between 1:30 and 3:00 p.m. The videos included at least one right and one left turn and objects including a pedestrian, an oncoming vehicle, a preceding vehicle, and a building. The practice video was about 1-min long and each experiment video was 3-min long.

The study was approved by the Institutional Review Board of Kyoto Institute of Technology. All participants agreed to the experimental procedure and protection of their data.

\subsubsection{Procedure}

Participants signed a consent form and completed a questionnaire about their driving experience. The experimenter provided a brief introduction and asked the participant to imagine that he/she was sitting in the driver's seat of an automated vehicle and to continue holding the wheel during the driving task. Participants watched the videos taken in Osaka and San Francisco after viewing the short practice video. The order of the experiment videos was counterbalanced to control for order effects. The participants were asked to complete a situational awareness questionnaire after each video. The eye tracker was calibrated before each video was presented. While the participant was watching the videos, the experimenter waited outside the room. The entire procedure took about $20 \mathrm{~min}$ to complete.

\subsubsection{Analysis}

To determine the direction of the participants gaze, each frame was divided into several areas based on the type of road (i.e., straight or curved). During a scene on a straight road, each frame was divided into seven parts: (a) the left lane, (b) the right lane, (c) the vehicle's lane, (d) above the middle, (e) buildings on the right, (f) buildings on the left, and (g) in the middle (Figure 2). When approaching a curve and waiting for an oncoming vehicle, each frame was divided vertically into three areas: the oncoming lane, the middle, and the direction in which the driver was moving. While turning right or left, each frame was vertically divided into three equal parts: the left, the center, and the right. Moreover, when the driver's eyes moved up or down while taking a curve, each frame was horizontally divided into two areas amounting to six areas in this scene. The method for dividing the frames was based on that of a previous study [9]. In each situation, when there was a specific object, such as a focal object or a pedestrian, the area of that object was defined according to the method of Harbluk and Noy [11].

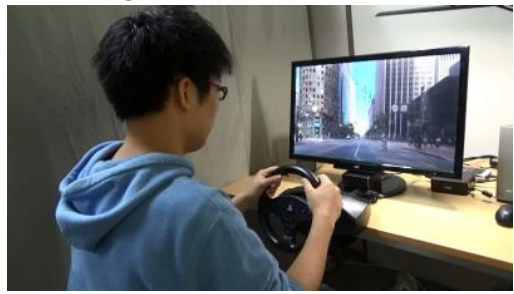

Figure 1: The experiment room

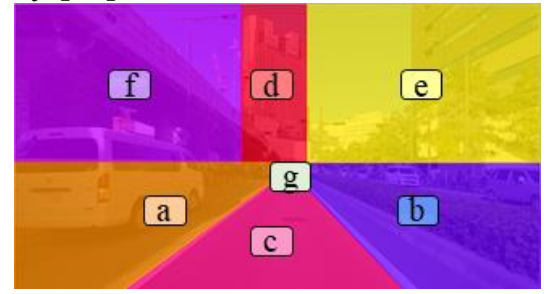

Figure 2: Example of a separated frame 


\subsection{Results}

Although one participant in the expert driver group had driven in the United States (U.S.) one time, the participant was not excluded from the study because we judged that 1 day was not enough to adjust to driving in the U.S.

\subsubsection{Total Fixation Duration in the Prescribed Areas}

Total fixation duration in the prescribed areas was calculated using a Tobii I-VT filter [13] that corresponded to the algorithm of Komogortsev et al. [14]. The I-VT fixation algorithm is a velocity-based classification algorithm [13][15]. Figure 3 shows the total fixation duration of the novice and expert drivers and the results of the two-samples $t$-tests.

The analysis of total fixation duration on the left lane area during a scene showing a straight road in Osaka revealed that the total fixation duration of expert drivers was significantly greater than that of novice drivers, $t(29.6)=1.95, p<0.05$. In the right lane and middle areas of the same scene, the total fixation durations of novice drivers were significantly longer than those of the expert drivers, $t(27.5)=1.75$ and $t(26.0)=2.26, p<0.05$, respectively. When turning right, the $t$-test revealed a marginally significant difference in total fixation duration on the area to the right, such that expert drivers gazed longer at that area than did novice drivers, $t(45)=1.39, p<$ 0.10 . When turning left, the difference in total fixation duration between groups was marginally significant with the novice drivers tending to focus longer on the area to the left than did expert driver, $t(29.1)=1.33, p<0.10$. In the same scenario, the $t$-test revealed a marginally significant difference in total fixation duration, indicating that the expert drivers gazed longer at a store with a large red sign than did novice drivers, $t(25.4)=1.64, p<0.10$.

During a scene showing a straight road in the San Francisco driving video, the $t$-test revealed a marginally significant difference in total duration of fixation on the area above the middle of the scene, $t(21.1)=1.41, p<0.10$. These results showed that expert drivers focused longer on this area than novice drivers did. In the same situation, expert drivers also exhibited significantly longer total fixation on buildings on the left, $t(27.0)=1.95, p<0.05$ (Figure 4).

No significant between-group differences in fixation were found for any other areas. Figure 5 shows the scenes where significant differences in total fixation duration appeared.

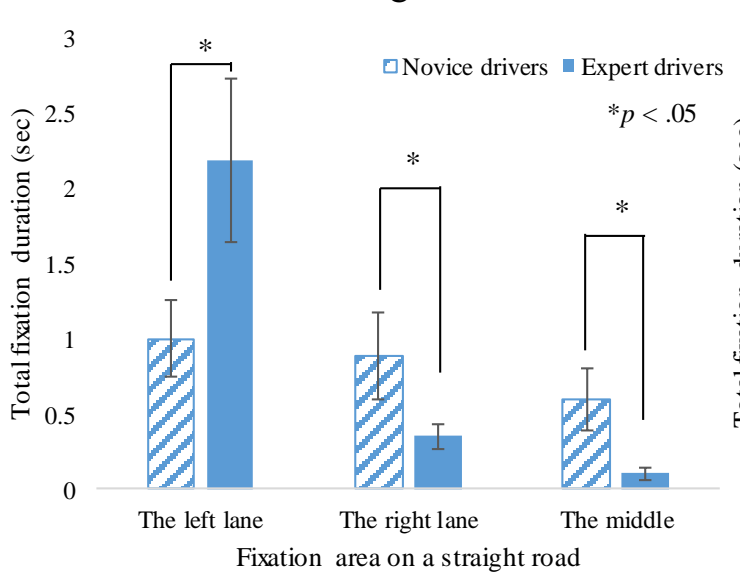

(a)

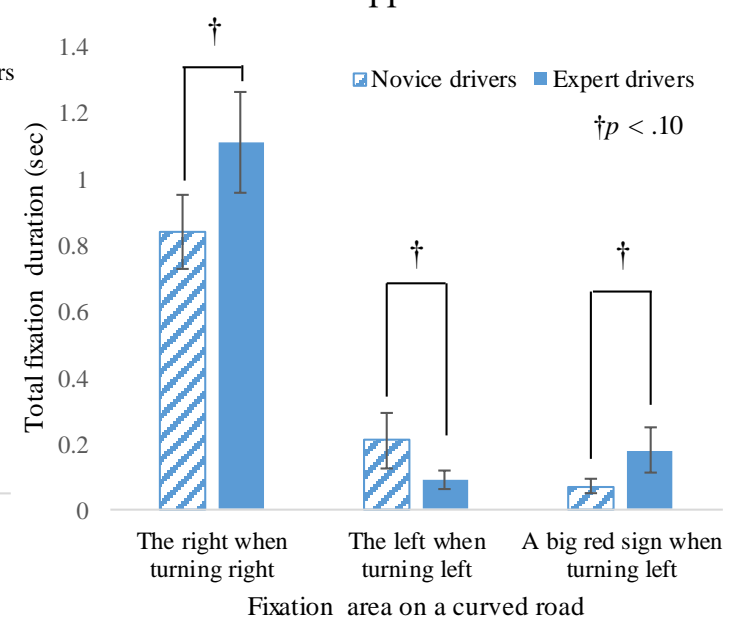

(b)

Figure 3: The mean total fixation duration of the novice and expert drivers in each of the prescribed areas in the Osaka driving video: (a) The mean total fixation duration on a straight road, (b) The mean total fixation duration on a curved road (The error bars represent standard errors). 


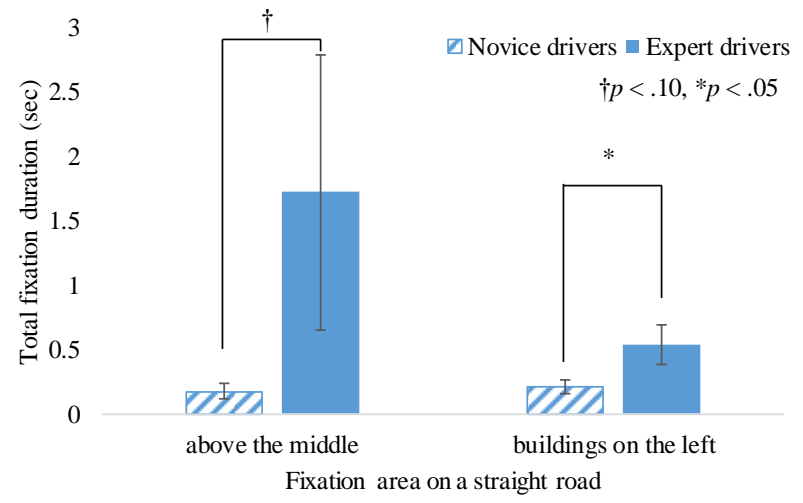

Figure 4: The mean total fixation duration of the novice and expert drivers in each of the presented areas in the San Francisco Driving video. (The error bars represent standard errors.)

(a)
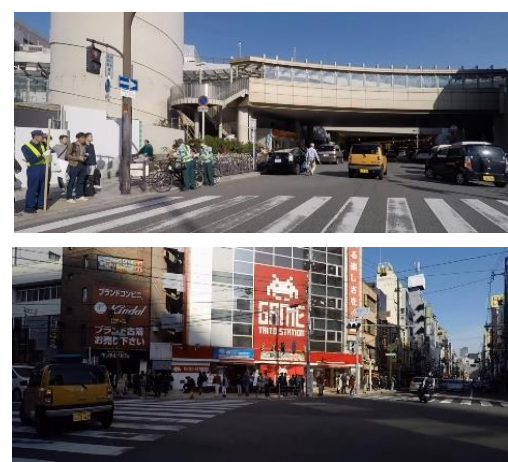

(b)

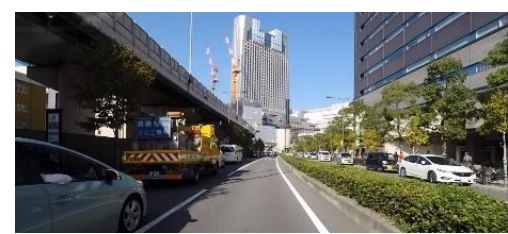

(d)

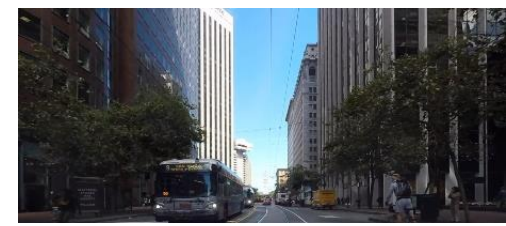

Figure 5: Sample frames in each situation where differences were indicated: (a) a straight road in Osaka, (b) a curved road in Osaka (right), (c) a curved road in Osaka (left), (d) a straight road in San Francisco.

\subsubsection{Fixation Duration During the Full Videos Without Divisions}

Additionally, total fixation duration was determined without distinguishing among AOIs in each driving video. Figure 6 shows the mean total fixation duration of novice and expert drivers during the videos. The one-sample $t$-test revealed that the fixation duration of expert drivers was marginally significantly different between the Osaka and San Francisco driving videos, such that fixation duration was shorter in the San Francisco video than in the Osaka video.

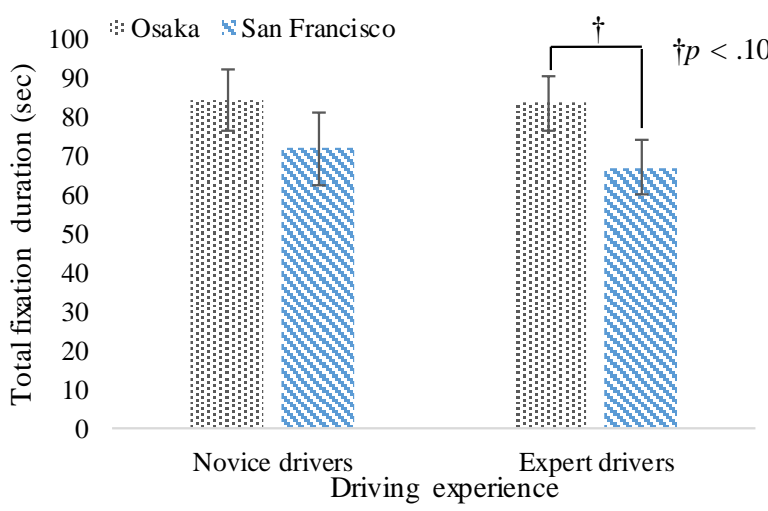

Figure 6: The mean total fixation duration of novice and expert drivers during the videos without division into areas and scenes. (The error bars represent standard errors.) 


\subsection{Assessing the Validity of the Experiment and Differences in Eye movements}

The differences in total fixation duration within the prescribed areas in the Osaka driving video were consistent with those reported previously [4][5][6]: novice drivers tended to gaze at a guardrail when on a straight road and tended to move their eyes from the center to the outside edge of the lane when driving on a curve. When driving on a straight road with a guardrail in the right lane area, novice drivers looked at the guardrail longer than did expert drivers. When turning right, the expert drivers focused on the right. When turning left, novice drivers gazed at the area on the left longer than did expert drivers. Moreover, we found that novice drivers focused on the middle of the road when driving on a straight road, which was consistent with the findings of a previous study showing that the fixation duration and fixation area of novice drivers were longer and narrower, respectively, than those of expert drivers [4].

Although these tendencies when driving on a curve were consistent with those of a previous study [6], they were not consistent in the two-curve driving scenarios. The responses on the situational awareness questionnaire may explain this inconsistency. In the right curve scenario, a pedestrian was walking on the left side in the area on the left. The situational awareness questionnaire for the Osaka video revealed that the percentage of correct answers about the pedestrian was not significantly different between the novice and expert drivers, suggesting that the pedestrian reduced the difference in total fixation duration for the area on the left. In the left curve scenario, a big red sign appeared in the center and right areas. The total fixation duration for the area around the sign showed that expert drivers tended to gaze at the area longer than did novice drivers. However, the mean percentage of correct answers for questions about the sign did not differ between experts and novices. These findings indicate that the expert drivers tended to gaze at the sign longer than novice drivers, although the sign attracted eye fixation regardless of driving experience. Therefore, it appears that the difference in degree of attention to the sign affected the total fixation duration for the area on the left while turning left. Thus, these findings support the validity of our study.

The comparison of total fixation duration on various areas between the San Francisco and Osaka driving videos revealed that the areas showing statistically significant differences in total fixation duration were completely different. In the straight road scene in the San Francisco video, total fixation times for the upper middle area and buildings to the left were significantly different between groups; expert drivers gazed at these areas longer than the novices. We found no difference in total fixation duration between these scenes. However, the analysis with no AOIs or scene divisions revealed that the total fixation duration of expert drivers was shorter in the San Francisco driving video than in the Osaka video, indicating that the expert drivers were more likely to be influenced by familiarity with the driving situation than novices. A previous study [8] found that increasing cognitive workload decreased fixation duration. Thus, expert drivers may need to think more while driving abroad than while driving in their own country owing to the difficulty in adapting to the new situation and overcoming regular patterns.

In conclusion, familiarity with the driving situation had a major impact on driver's eye movements, and the driving situation had a greater effect on expert drivers than on novice drivers due to the increased cognitive workload.

\section{Additional Analyses of Gaze Point and Saccades}

\subsection{Gaze Point on a Curve}


Shinohara and Nishizaki [10] measured fixation duration in prescribed areas and concluded that the disparity between their findings for left curves and those of a previous study [8] could be explained by the presence of a big red sign, which attracted the gaze of the drivers. In this section, we evaluate the validity of that conclusion.

Figure 7 the mean horizontal gaze position of novice and expert drivers while turning right. The horizontal axis indicates the frames where participants returned their gaze from the inside edge of the lane to the center $(1.2 \mathrm{~s})$. The vertical axis shows the horizontal gaze position from 0 $\mathrm{mm}$ (left end of the frame) to $173 \mathrm{~mm}$ (center of the frame). The two solid lines show the mean horizontal gaze positions of the novice and expert drivers, respectively. The dashed lines are simple regressions of the mean horizontal gaze positions.

Simple linear regressions were calculated to assess between-group differences in the velocity of eye movements when drivers returned their gaze to the center of the lane. Significant regression equations were found for the novice drivers $\left(p<.001, R^{2}=0.765\right.$, adjusted $\left.R^{2}=0.761\right)$ and expert drivers $\left(p<.001, R^{2}=0.909\right.$, adjusted $\left.R^{2}=0.908\right)$. The regression coefficient for the novice drivers was $0.735(t(75)=15.6, p<.001)$ and that for the expert drivers was $1.56(t(75)=27.3, p<.001)$.

These findings suggest that the inconsistent findings for the left area was attributable to differences in the velocities with which the novice and expert drivers returned their gaze to the center.

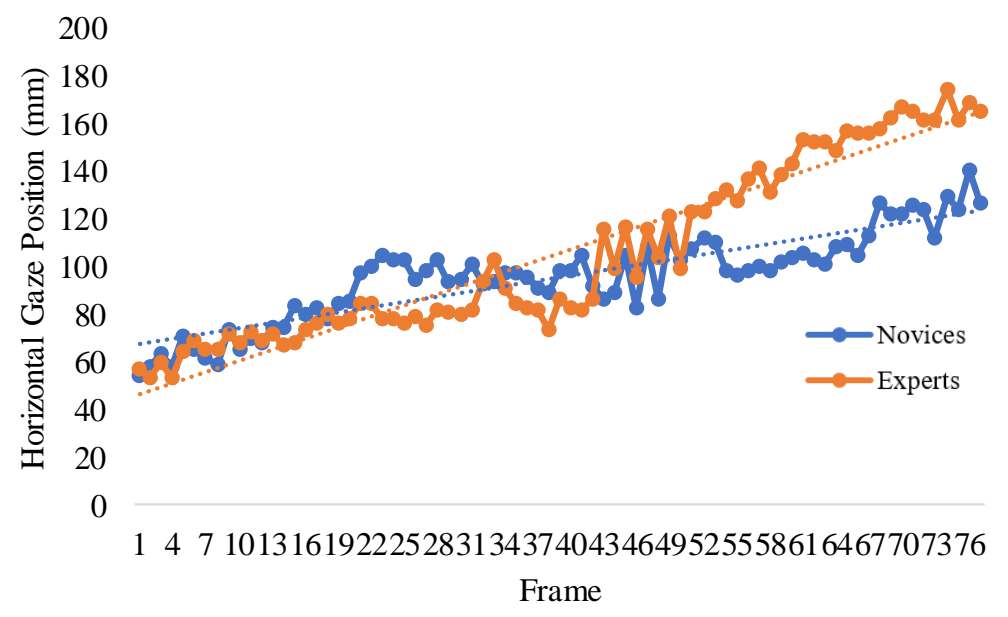

Figure 7: The mean horizontal gaze position of novice and expert drivers while turning right.

\subsection{Mean Number of Saccades}

Saccades are rapid ballistic movements of the eyes that change the point of fixation. We used a Tobii I-VT filter to identify saccades in the eye-tracking data. The number of saccades initiated in each driving video was calculated, and a $t$-test was used to compare the mean number of sac-cades initiated by novice and expert drivers. We found a significant between-group difference in the mean number of saccades in the San Francisco driving video $(t(45)=-2.76, p<0.01)$, whereas the mean number of saccades in the Osaka video was not significantly different between the novice and expert drivers. Figure 8 shows the mean number of saccades in the novice and expert groups for the San Francisco driving video. 


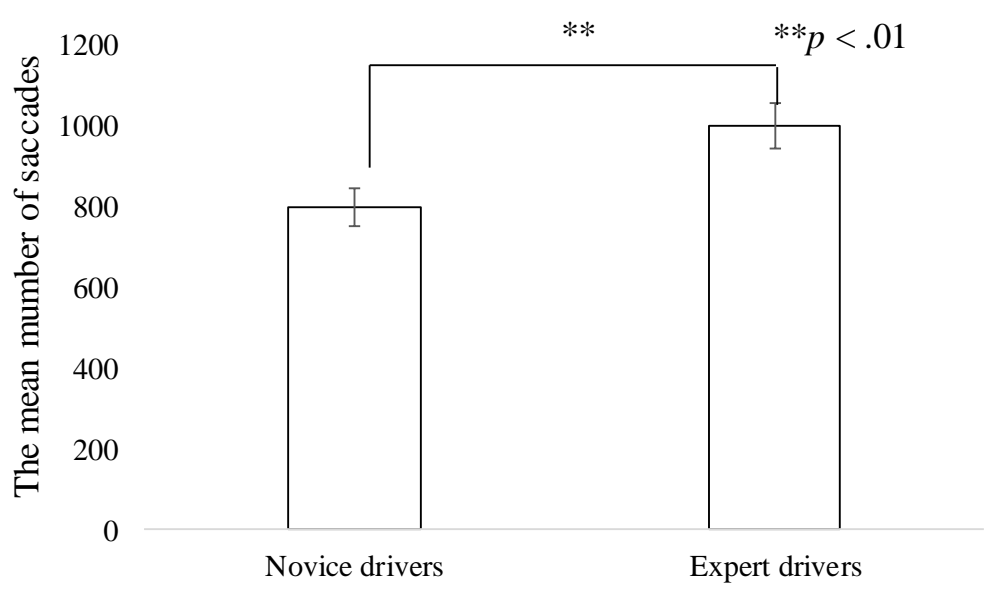

Figure 8: The mean number of saccades in the novice and expert groups for the San Francisco driving video. (The error bars represent standard errors.)

Figure 9 shows the cumulative average of the number of saccades in each frame for novice and expert drivers. The horizontal axis represents frames during the entire video ( $3 \mathrm{~min}$ ). Simple linear regressions were calculated to assess differences in increasing ratio of the number of saccades between novice and expert drivers. Significant regression equations were found for the Osaka driving video (novice drivers: $p<.001, R^{2}=0.999$, adjusted $R^{2}=0.999$; expert drivers: $p<.001, R^{2}=1.000$, adjusted $R^{2}=1.000$ ). The regression coefficient for novice drivers was $0.084(t(10804)=3720.6, p<.001)$ and that for expert drivers was $0.087(t$ $(10804)=9075.7, p<.001)$. Furthermore, significant regression equations were found for the San Francisco driving video, (novice drivers: $p<.001, R^{2}=1.000$, adjusted $R^{2}=1.000$; expert drivers: $p<.001, R^{2}=0.999$, adjusted $\left.R^{2}=0.999\right)$. The regression coefficient for novice drivers was $0.072(t(10832)=6019.4, p<.001)$, whereas that of the expert drivers was $0.092(t(10832)=4148.6, p<.001)$.

We found a significant between-group difference in the mean number of saccades emitted in the San Francisco video, but not in the Osaka video: novice drivers initiated significantly fewer saccades in the San Francisco video than did expert drivers. Previous studies have shown that an increased cognitive workload reduced the number of saccades [11][12], suggesting that the cognitive workload was greater for the novice drivers than for the expert drivers when viewing the San Francisco driving video. However, this finding is not consistent with the conclusion of Shinohara and Nishizaki [10] who argued that the longer fixation duration exhibited by expert drivers viewing the San Francisco video was due to increased cognitive workload.

The $R^{2}$ values strongly support the validity of the linear regression equations, indicating that the mean number of saccades increased linearly. This suggests that the saccades were not influenced by road type (i.e., straight or curved), or background (e.g., the number of buildings or pedestrians). Thus, it is reasonable to suggest that the significant difference between novice and expert drivers observed with the San Francisco driving video was related to familiarity with the driving situation because we found no significant difference between groups with the Osaka video. However, we could not directly compare the findings of each driving video due to differences such as the number of pedestrians. Thus, further study is needed to determine the main factors underlying the significant difference in the number of saccades.

Although our findings do not confirm that cognitive workload was a major contributing factor to the differences in total fixation duration, they do suggest that familiarity with the driving situation affects saccadic eye movements. 


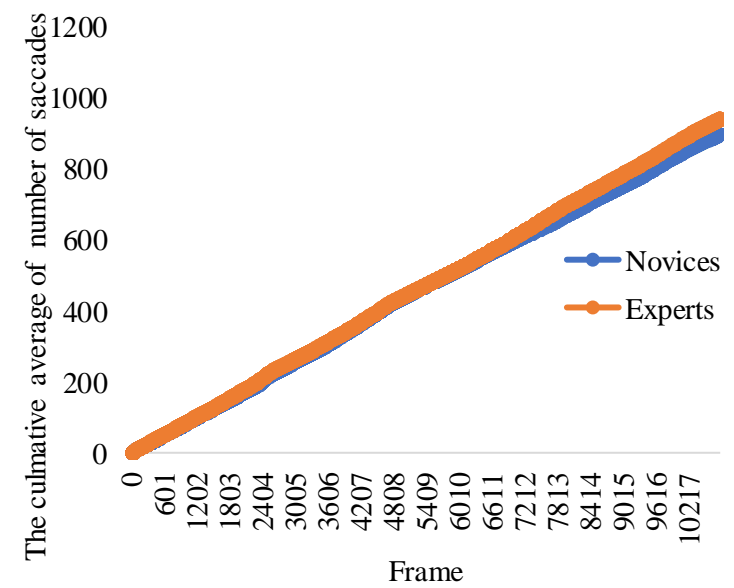

(a)

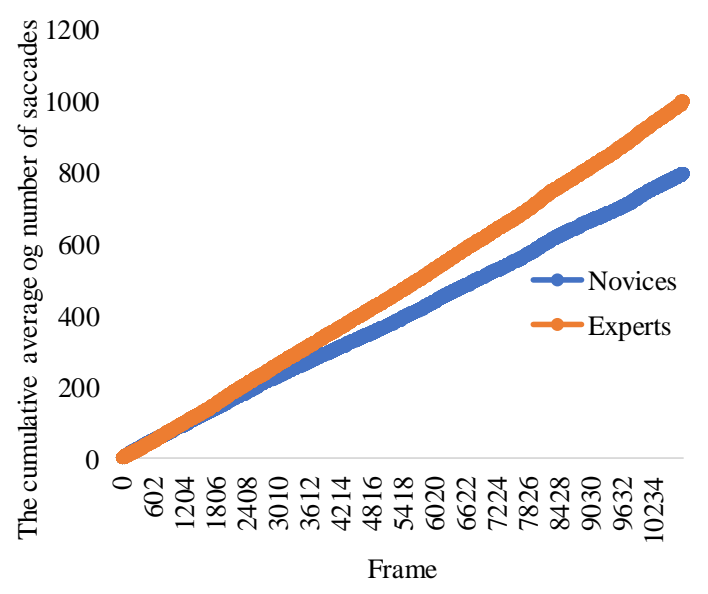

(b)

Figure 9: The cumulative average of the number of saccades in each frame for novice and expert drivers in each frame during each driving video: (a) the Osaka driving video, (b) the San Francisco driving video.

\section{Conclusions}

The findings of gaze position when turning left in the Osaka driving video support the validity of our study. Shinohara and Nishizaki [10] attributed the inconsistency with the findings of Miura [8] to an outstanding object. However, we showed that the inconsistency was the result of their analysis method, in which they divided frames into AOIs, and that when turning a curve, expert drivers returned their gaze to the center of the road more quickly than did novice drivers.

The between-group differences in the mean number of saccades suggested an effect of familiarity with the driving situation. When viewing the San Francisco driving video, an unfamiliar situation elicited fewer saccades in novice drivers than in expert drivers, whereas no difference in the number of saccades was observed for a familiar situation in the Osaka driving video. Moreover, the linear increase in the number of saccades suggests that increase ratio was related to familiarity with the driving situation. However, the relationship between driving in an unfamiliar situation and increased cognitive workload is not clear.

Automated driving systems should be designed to promote safe driving based on driver experience and background. Our findings support the development of automated driving systems that attend to drivers' characteristics; for example, a driver support system that evaluates eye movements and alerts the driver when the risk of a collision increases.

Directions for future studies include a more precise analysis of gaze position and identifying the factors underlying the differences in eye movements.

\section{Acknowledgement}

We thank the researchers at the Center for Design Research at Stanford University for helping to film the driving videos in San Francisco. We thank Prof. Hochin, Kyoto Institute of Technology, for his guidance and advice regarding analyses. 


\section{References}

[1] NHTSA, "National Motor Vehicle Crash Causation Survey: Report to Congress," 2008.

[2] H. Erwin, "Driver vision requirements," Society of Automotive Engineers Technical Paper Series, vol. 700392, 629-630, 1970,

[3] SAE On-Road Automated Vehicle Standards Committee. Taxonomy and Definitions for Terms Related to On-Road Motor Vehicle Automated Driving Systems; Technical Report J3016_201401; SAE: Hong Kong, China, 2014.

[4] R.R. Mourant, T.H. Rockwell, "Strategies of Visual Search by Novice and Experienced Drivers," Human Factors: The Journal of the Human Factors and Ergonomics Society, 1972, $14-4,325-335$

[5] K. Satoh, "Visual Search and Peripheral Vision Performance by Novice and Experienced Drivers," IATSS Review, 19-3, 1993.

[6] O. Laya, "Eye Movements in Actual and Simulated Curve Negotiation Task," ITASS Research, 16(1), 15-26, 1992.

[7] P.R. Chapman, G. Underwood, "Visual Search of Driving Situations: Danger and Experience," Perception, vol. 27, 951-964, 1998.

[8] T. Miura, "Active Function of Eye Movement and Useful Field of View in a Realistic Setting," In R. Gorner, G.d'Ydewalle \& R.Parham (Eds.), From Eye to Mind: Information Acquisition in Perception, Search and Reading, Amsterdam, 119-127, 1990.

[9] R. Paeglis, K. Bluss, A. Atvars, "Driving Experience and Special Skills Reflected in Eye Movements," Proc. of SPIE, 8155, 815516-1, 2011.

[10] Y. Shinohara, Y. Nishizaki, "Where do drivers look when driving in a foreign country?," Studies in Computational Intelligence, 2017, 721, 151-164.

[11] J.L. Harbluk, Y.I. Noy, "The Impact of Cognitive Distraction on Driver Visual Behaviour and Vehicle Control," report no. TP13889E. Transport Canada, Ontario, 2002.

[12] Y. Dong, Z. Hu, K. Uchida, N. Murayama, "Driver Inattention Monitoring System for Intelligent Vehicles: A Review," IEEE Transactions on intelligent transportation systems, 2011, 12, 2 .

[13] A. Olsen, “The Tobii I-VT fixation filter: Algorithm description,” Tobii Technology, 2012.

[14] O.V. Komogortsev, D.V. Gobert, S. Jayarathna, D.H. Koh, S.M. Gowda, "Standardization of Automated Analyses of Oculomotor Fixation and Saccadic Behaviors," IEEE Transactions on biomedical engineering, 2010, 57, 11 .

[15] D.D. Salvucci, J. H. Goldberg, "Identifying fixations and saccades in eye-tracking protocols," Proc. of the symposium on Eye tracking research and applications, Palm Beach Gardens, Florida, United States, 2000, 71-78. 